\title{
Ge/Si Quantum Dots Superlattices Grown at Different Temperatures and Characterized by Raman Spectroscopy and Capacitance Measurements
}

\author{
A. D. Rodrigues, ${ }^{1}$ A. J. Chiquito, ${ }^{1}$ G. Zanelatto, ${ }^{1}$ A. G. Milekhin, ${ }^{2}$ \\ A. I. Nikiforov, ${ }^{2}$ V. V. Ulyanov, ${ }^{2}$ O. P. Pchelyakov, ${ }^{2}$ D. R. T. Zahn, ${ }^{3}$ and J. C. Galzerani ${ }^{1}$ \\ ${ }^{1}$ Department of Physics, Federal University of São Carlos, CP 676, 13565-905 São Carlos, SP, Brazil \\ ${ }^{2}$ Institute of Semiconductor Physics, Novosibirsk 630090, Russia \\ ${ }^{3}$ Institut für Physik, Technische Universität Chemnitz, 09107 Chemnitz, Germany
}

Correspondence should be addressed to A. D. Rodrigues, ariano@df.ufscar.br

Received 28 March 2012; Accepted 31 July 2012

Academic Editor: Michael C. Tringides

Copyright (C) 2012 A. D. Rodrigues et al. This is an open access article distributed under the Creative Commons Attribution License, which permits unrestricted use, distribution, and reproduction in any medium, provided the original work is properly cited.

\begin{abstract}
Ge/Si heterostructures with Ge self-assembled quantum dots (SAQDs) grown at various temperatures by molecular beam epitaxy were investigated using resonant Raman spectroscopy and capacitance measurements. The occurrence of quantum confinement effects was confirmed by both techniques. For the structures grown at low temperatures $\left(300-400^{\circ} \mathrm{C}\right)$, the SAQDs optical phonon wavenumbers decrease as the Raman excitation energy is increased; this is an evidence of the scattering sensitivity to the size of the SAQDs and to the inhomogeneity in their sizes. However, the opposite behavior is observed for the SAQDs grown at higher temperatures, as a consequence of the competition between the phonon localization and internal mechanical stress effects. The $E_{1}$ electronic transition of the Ge in the SAQDs was found to be shifted towards higher energies as compared to bulk Ge, due to biaxial compressive stress and to the electronic confinement effect present in the structures. The intermixing of Si atoms in the quantum dots was found to be much more significant for the sample grown at higher temperatures. The capacitance measurements, besides confirming the existence of the dots in these structures, showed that the deepest Ge layers lose their 0D signature as the growth temperature increases.
\end{abstract}

\section{Introduction}

Undoubtedly, Ge/Si self-assembled quantum dots (SAQDs) present an interesting technological potential, confirmed by several studies pointing to the development of devices such as photodetectors operating in the midinfrared [1], transistors [2], memory devices [3], nonlinear optic devices [4], and lasers [5], based in these structures. It is obvious that for the production of these devices it is necessary that the growth techniques are fully controlled and that the characteristics of the obtained material are very well known. In comparison with single-layered structures, the quantum dots superlattices present superior potential for optoelectronic applications due to the larger volumetric density of islands, the modified confining potential, and the suppression of surface recombinations. Recently electroluminescence in frequencies of order of $\mathrm{THz}$ was observed in $\mathrm{Si} / \mathrm{Ge}$ superlattices [6]. Nevertheless, in system in which the Ge islands are embedded into a $\mathrm{Si}$ matrix, besides the nominal thickness of the $\mathrm{Ge}$ layers, the growth temperature strongly influences both the size and the chemical composition of islands, due to SiGe intermixing [7-11]. The growth dynamics in a multilayer structure can be fundamentally different as compared to the monolayer case. During the process of preparation, the sample temperature is successively varied in order to establish ideal conditions for the growth of either $\mathrm{Si}$ or $\mathrm{Ge}$ layers. So, the deeper the Ge layer is, the most prominent can be the effect of intermixing, that directly induces modifications in the electronic energy spectrum. In this work we have combined Raman spectroscopy and capacitance measurements in order to study the compositional structure and the growth dynamics of Ge/Si SAQDs superlattices 


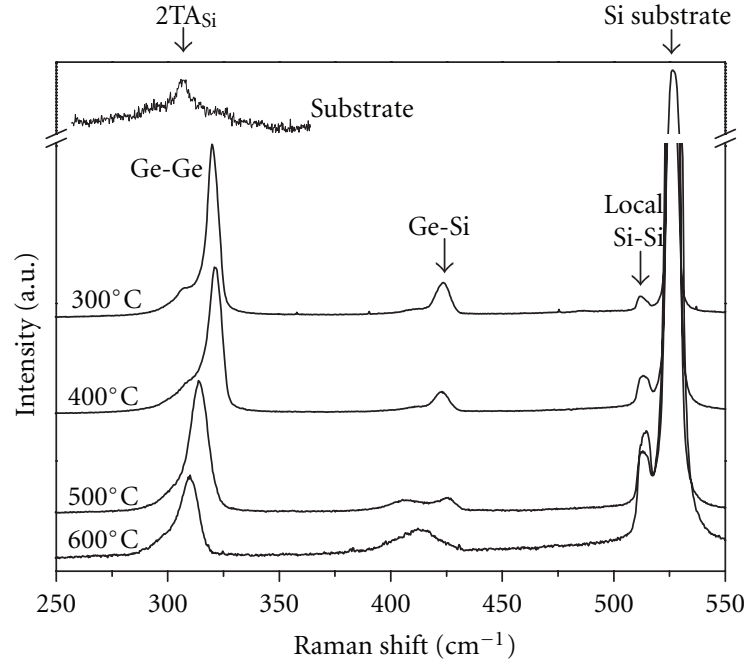

FIGURE 1: Raman spectra of the samples grown at several temperatures, measured at $8 \mathrm{~K}$ and excited with the $514.5 \mathrm{~nm}$ laser line.

grown at different temperatures. By obtaining the resonant Raman scattering profiles, we could draw some conclusions about the electronic properties of the samples as a function of growth temperatures. Furthermore, since the vibrational properties play an important role both in the charge and in the heat transport, the knowledge of the Raman spectra of these nanostructures is fundamental for their applications in devices.

\section{Experimental}

The samples were grown by Molecular Beam Epitaxy (MBE) following the Stranski-Krastanov regime, on (001) Si substrates. The set of four samples consists of alternate $\mathrm{Si}$ and Ge layers with nominal thicknesses $500 \AA$ and 10 monolayers, respectively, repeated five times, ending with $\mathrm{Si}$. The growth temperature was varied, assuming the values 300, 400, 500, and $600^{\circ} \mathrm{C}$. An investigation using cross-sectional highresolution transmission electronic microscopy (HRTEM) of samples similarly prepared [12] shows that, for the here used growth parameters, SAQDs are expected with dimensions that increase with the growth temperature: the average base sizes vary between 10 and $100 \mathrm{~nm}$ and the dot heights vary from 2 to $6 \mathrm{~nm}$. Resonant Raman scattering was accomplished with the excitation given by two sources: a Rhodamine 6G dye laser and an $\mathrm{Ar} / \mathrm{Kr}$ laser. For all measurements the power density was kept below $2 \times 10^{5} \mathrm{~W} / \mathrm{m}^{2}$ in order to prevent sample heating. A triple monochromator T64000 equipped with a liquid nitrogen cooled CCD detector was then used for the analysis of the scattered light. The spectra were taken at the temperature $8 \mathrm{~K}$.

For the capacitance experiments, an $\mathrm{Au}_{0.5} \operatorname{In}_{0.5}$ alloy was used as the ohmic contact (annealed at $450^{\circ} \mathrm{C}$ for $120 \mathrm{~s}$ ) and Au was evaporated in order to obtain the Schottky one (200 $\mathrm{nm}$ thick layer with $500 \mu \mathrm{m}$ diameter). The measurements were realized with conventional $\mathrm{AC}$ techniques, the signal detection being obtained with a lock-in amplifier. These measurements were taken at 300 and $150 \mathrm{~K}$, with $10 \mathrm{mV}$ and $100 \mathrm{kHz}$ as the excitation voltage and excitation frequency, respectively.

\section{Results and Discussions}

We present the unpolarized Raman spectra of the studied samples, grown at different temperatures, in Figure 1. All the spectra exhibit strong peaks in the interval $310-320 \mathrm{~cm}^{-1}$. Another feature around $420 \mathrm{~cm}^{-1}$, together with a shoulder in the low-energy region, could be observed for the samples grown at 300,400 , and $600^{\circ} \mathrm{C}$; in the case of the sample grown at $500^{\circ} \mathrm{C}$ the presence of two peaks is evident in this region of energies. The lower-energy peaks present in our spectra can be attributed to the Ge-Ge vibration. In bulk materials this mode appears at $304 \mathrm{~cm}^{-1}$; for our samples however this peak was found to be blue shifted. Together with them it can be observed low-energy shoulders corresponding to the second-order $2 \mathrm{TA}_{\mathrm{Si}}$. The spectrum of the substrate in this interval (shown for comparison in the top of the figure) confirms this assertion. The features around $420 \mathrm{~cm}^{-1}$ can be associated to the optical vibrations of the Ge-Si alloy present in the grown structures [13]. The frequencies of the optical vibrational modes of both Ge-Ge and Ge-Si alloy depend on two concurrent effects: the interdiffusion strength of the $\mathrm{Si}$ atoms through the Ge layers_-provoking blue shifts-and the confinement of the optical phonon inside the SAQDs_causing a red shift due to the negative phonon dispersion relation. The resonant Raman scattering, detailed below will help us to drive some conclusions about the competition between these effects. Finally, we can notice around $525 \mathrm{~cm}^{-1}$ the Si bulk phonon, originated at the (001) substrate and around $512 \mathrm{~cm}^{-1}$, a feature with lower intensity, that can be attributed to a local Si-Si mode [13]. Figure 2 presents the profiles of the resonant Raman scattering of the Ge-Ge vibration for the four samples. For each spectrum the Ge-Ge signal was normalized with respect to the Si signal of the substrate and plotted as a function of the excitation energy. The most intense maxima appearing in the four curves are attributed to the $E_{1}$ electronic transition of Ge. At room temperature, this transition for bulk Ge occurs at $2.23 \mathrm{eV}$ [14]. However, both the electronic confinement and the compressive stress of the islands can contribute to the blue shifting in $E_{1}$ energy. The observed values of the $E_{1}$ transition agree with the data obtained by Kwok et al. [15]. In the case of the samples grown at 400,500 , and $600^{\circ} \mathrm{C}$, an additional increase in the GeGe signal intensity is observed, for excitation energies 2.54, 2.52 , and $2.68 \mathrm{eV}$, respectively. We believe that the electronic transitions of the $\mathrm{Ge}_{x} \mathrm{Si}_{1-x}$ alloy, present in the samples, are responsible for these features. As a matter of fact, Cerdeira et al. [16] reported resonances in this energy range for particular stoichiometries of the referred alloy. In principle, the electronic transitions of the GeSi alloy are not expected to interfere in the resonance of the Ge-Ge optical modes. However, considering that these modes are not rigorously confined in the regions of pure Ge of the islands (in other 


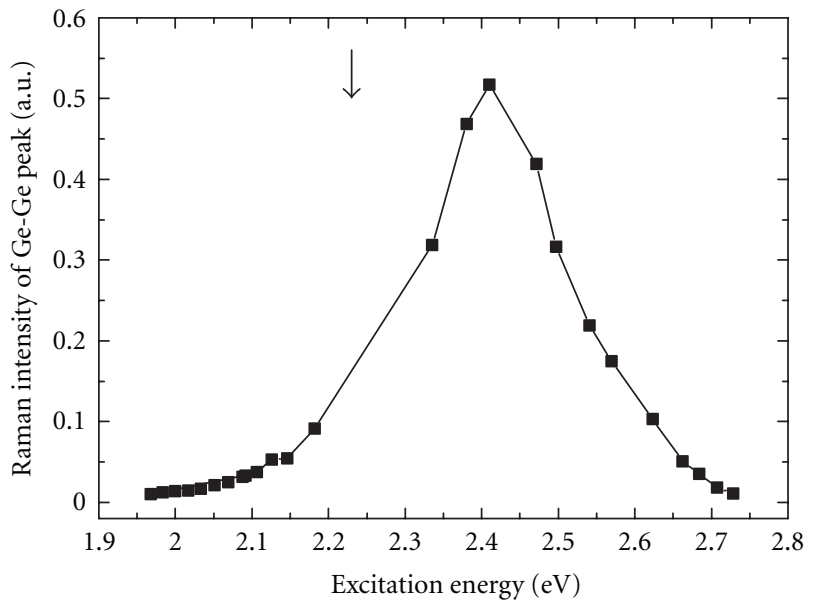

(a)

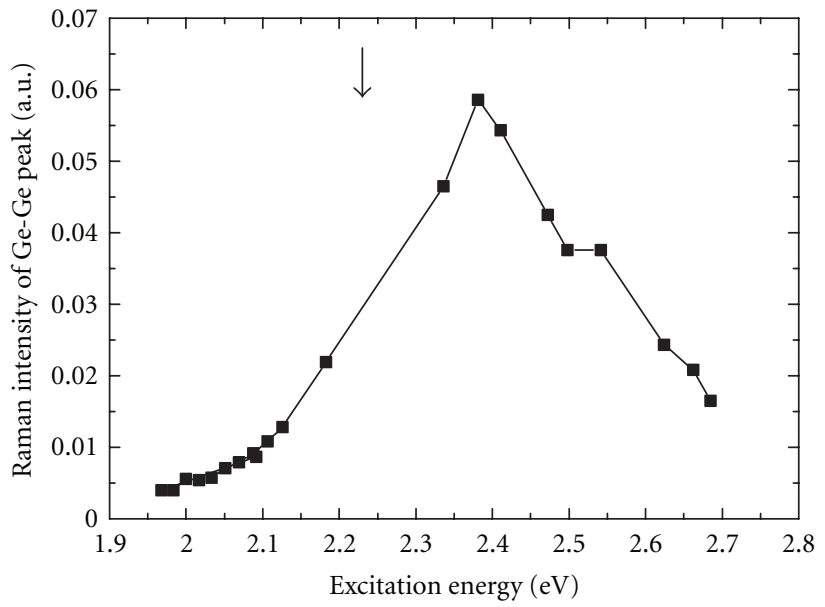

(c)

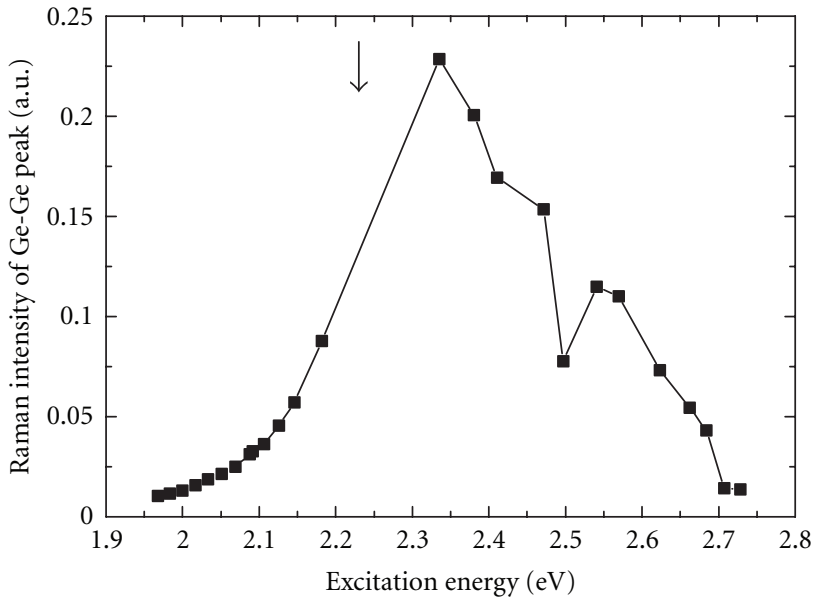

(b)

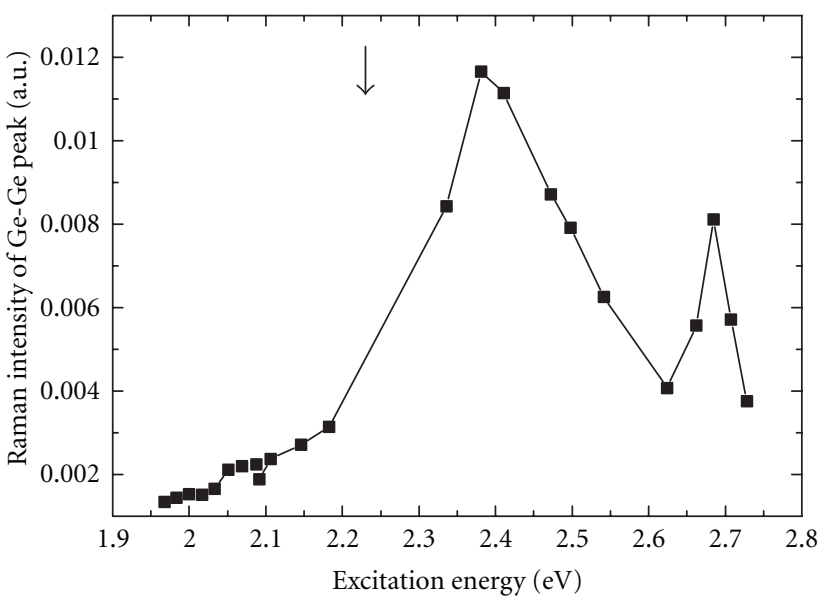

(d)

FIgure 2: The intensity of the Ge-Ge signal as a function of the excitation energy for the samples grown at (a) $300^{\circ} \mathrm{C},(\mathrm{b}) 400^{\circ} \mathrm{C},(\mathrm{c}) 500^{\circ} \mathrm{C}$, and $(\mathrm{d}) 600^{\circ} \mathrm{C}$. The arrows show the bulk Ge resonance.

words, the vibration relative to these phonons can extend as far as the first atomic layers of the GeSi alloy), it is quite reasonable to suppose that the specific Ge vibrations have enough amplitudes in the alloy in order to couple with its electrons and consequently contribute to the resonance curve of the Ge optical mode, a "signature" of the electronic states of the alloy. Influences of the GeSi alloy in the intensity of the Ge-Ge mode were also observed in Ge/Si superlattices [17], where the electronic transition was attributed to the $E_{1}$ gap of a $\mathrm{Ge}_{0.5} \mathrm{Si}_{0.5}$ alloy. The sample grown at $300^{\circ} \mathrm{C}$ does not show this transition, as observed from Figure 2(a). This fact indicates a negligible Si content in the islands, due to the low diffusion rate of $\mathrm{Si}$ towards the Ge layers. Further referring to Figure 2, it is also quite remarkable the decrease in the intensities of the resonance curves as the growth temperature increases. This can be associated to the density of the quantum dots in the samples. Since the monitored GeGe signal originates mainly from the SAQDs, the larger the number of dots per area is, the stronger the phonon signal should be and consequently the resonance curve intensity. So we can definitely affirm that the SAQDs density decreases as the growth temperature increases; at $600^{\circ} \mathrm{C}$ we are probably close to a two-dimensional GeSi layer, as confirmed below by the $\mathrm{C}-\mathrm{V}$ measurements. Figure 3 shows the optical phonon wavenumber of the Ge SAQDs as a function of the excitation energy. Here again we should be careful in the analysis of the results, since we are dealing with the competition between two phenomena: for the SAQDs of smaller sizes the optical phonons confinement effect would contribute to the frequency of the Ge-Ge vibrational mode, while for the SAQDs of larger sizes the confinement effect could be neglected and the intrinsic stress will prevail in the frequency shift determination. Raman scattering of a size distribution of SAQDs takes place in a highly selective way. Since the quantum confinement is stronger (and, consequently, the electronic transitions are more energetic) in the smallest dots, the higher the excitation energy is, the stronger the contribution of these structures to the Raman spectrum is (which is than dominated by them). For the same reason, for the lowest excitation energies, almost the totality of 


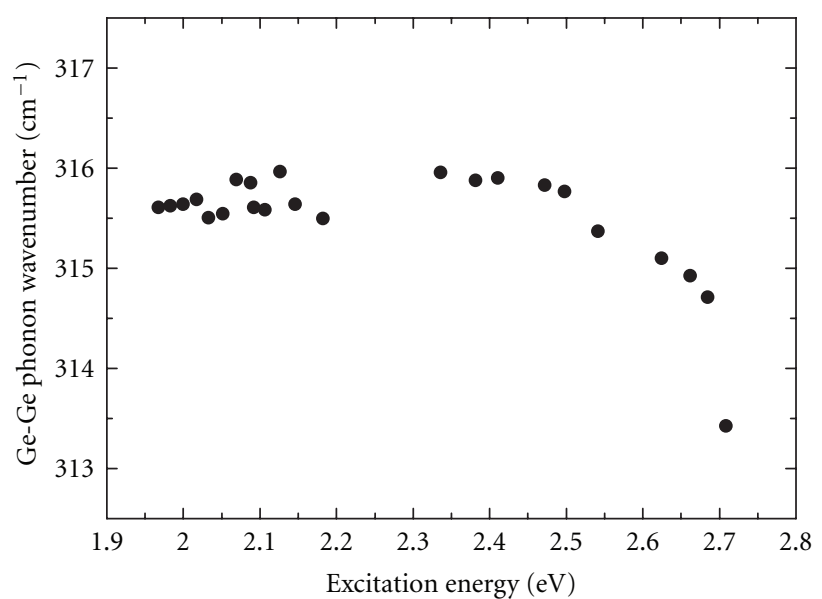

(a)

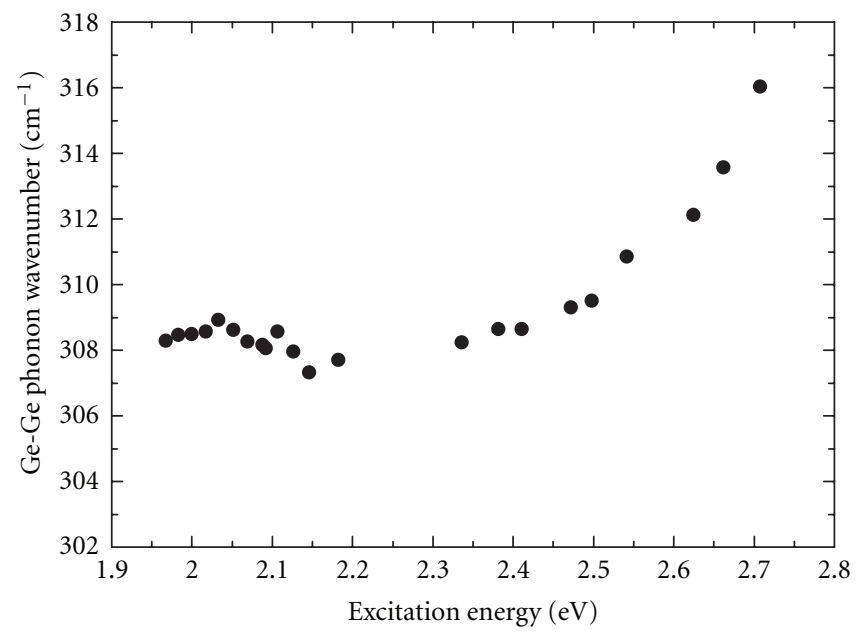

(c)

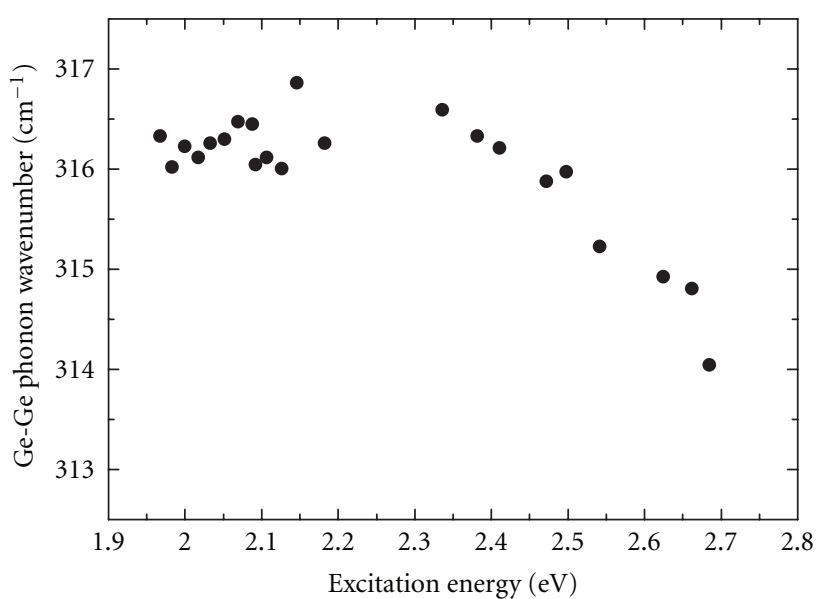

(b)

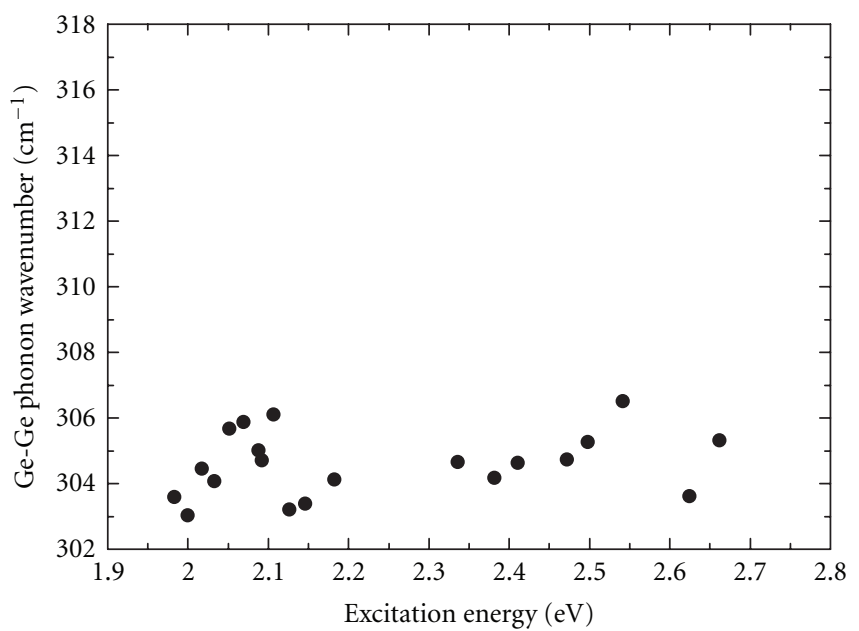

(d)

FIgURE 3: The quantum dot optical phonon wavenumbers as a function of the excitation energy for the four samples grown at (a) $300^{\circ} \mathrm{C}$, (b) $400^{\circ} \mathrm{C}$, (c) $500^{\circ} \mathrm{C}$, and (d) $600^{\circ} \mathrm{C}$.

the spectrum intensity will be originated from the SAQDs of larger dimensions, because these energies will be insufficient to excite electronic states with higher energies, like those of the smallest dots. Besides, the larger the dimension of the quantum dot is, the less important the confinement effect of its optical phonon will be. As a consequence, in a sample of this kind, the tuning of the excitation energy would select the dimensions of the quantum dots that more effectively contribute to the Raman scattering. If we refer to the sample grown at $300^{\circ} \mathrm{C}$ (Figure 3(a)), we observe that a change in the energy of the optical phonon occurs for excitation energies higher than $2.5 \mathrm{eV}$. The shift to lower wavenumbers-due to the confinement effect-is typical of materials presenting a negative dispersion of phonons, such as Ge; as expected, the confinement progressively increases with the photon energy, clearly indicating the existence of a size distribution of the Ge SAQDs for this growth temperature. Considering now the sample grown at $400^{\circ} \mathrm{C}$ (Figure 3(b)), for the energy range $(1.95-2.35 \mathrm{eV})$ in which the confinement effect is not relevant, the phonon wavenumber is slightly higher as compared to the former sample indicating that here the quantum dots dimensions have increased. The curve decays in a more smooth way, showing that the size distribution is now more heterogeneous. The sample grown at $500^{\circ} \mathrm{C}$ presents an interesting behavior, opposite to the first two ones. We believe that the SAQDs are here distributed in two distinct regimes, as observed before by Milekhin et al. [18] for samples where a thin $\mathrm{SiO}_{2}$ layer was grown over the Si substrates. In the first regime, the three-dimensional islands have much larger dimensions than those found in the first two samples, such that here the compressive stress is lower, as observed before for this kind of sample [12]; being so, for the lower excitation energies utilized, where these SAQDs are responsible for the scattering, the optical phonon wavenumber is quite near those expected for the bulk Ge. We can say that these islands have lost their zero-dimension signature, although the three-dimensional growth may still occur. In the second regime, the SAQDs' dimensions are comparable to those observed in the first two samples. Consequently, they conserve part of the wetting 
layer compressive stress, while being subjected to the phonon confinement effect. It is observed that, as the excitation energy increases, approximating the characteristic electronic transitions of these smaller SAQDs, such structures are the major responsible for the scattering, such that the optical phonon wavenumbers are quite similar to those measured in the former samples when excited with lower energies. This is an evidence that the smallest SAQDs in the sample prepared at $500^{\circ} \mathrm{C}$ would present dimensions close to those found in the larger islands grown at lower temperatures $\left(300\right.$ and $\left.400^{\circ} \mathrm{C}\right)$. The existence of two distinct regimes in the size distribution of the SAQDs can still be observed in another interval of the Raman spectrum of the sample grown at $500^{\circ} \mathrm{C}$. In the region around $420 \mathrm{~cm}^{-1}$, it is evident the presence of two separate peaks related to optical vibrations in the GeSi alloy. It is known that the phonon energies in binary alloys are influenced by the amount of each chemical element forming the compound. Ratto et al. [11] demonstrated that the alloying process is strongly dependent on the areas of the islands' bases, that is, the larger the SAQDs dimensions are, the more prominent the Si intermixing through the Ge quantum dots will be. Being so, we expect that the alloying process is more effective in the SAQDs of the first regime (thus producing a Si richer alloy), in comparison to those of the smaller islands. This originates Raman peaks with different energies: a more energetic one, related to the Si richer alloy present in the largest SAQDs, and a lower energetic one, due to the scattering in the smallest islands (in which the alloying process is less efficient). Considering now Figure 3(d), we observe that the Ge optical mode shows no relevant variation with the excitation energy indicating that the highest growth temperature possibly gave origin to very large three-dimensional GeSi islands. In this case the Ge content in the GeSi SAQDs is about 53\% [12]. As a matter of fact, we should recall that for this sample we observed the alloy $E_{1}$ resonance at $2.68 \mathrm{eV}$ (Figure 2(d)); it should also be observed that, in [17], in the case of the $\mathrm{Ge}_{0.5} \mathrm{Si}_{0.5}$ solid solution, it was claimed that the $E_{1}$ resonance appears at $2.7 \mathrm{eV}$. As a consequence of the light attenuation through the sample and since we are dealing with a superlattice with five periods, it is expected a major contribution of shallower layers to the information taken from the analyses of Raman spectra. In order to characterize the morphology of deeper layers, we have performed $\mathrm{C}-\mathrm{V}$ measurements. Under zero field, the depletion layer is around $2000 \AA$ thick. As a result, through this technique, we could access specifically the deepest two Ge layers. Figure 4 depicts the $\mathrm{C}-\mathrm{V}$ measurements for the samples grown at 300 and $400^{\circ} \mathrm{C}$, respectively. In these heterostructures, the capacitance is caused by two distinct contributions: a three-dimensional capacitance due to the electronic distribution in the volume around the quantum region and the capacitance due to the quantum confined electrons [19]. The contribution of the quantum dots to the total capacitance is a direct consequence of their characteristic density of states and their occupation at a given temperature. Taking into account that the samples were undoped the expected electron density at the SAQDs were very low: using the well-known Schottky capacitance theory $[20,21]$, the electron density was found to be $6.0 \times 10^{12} \mathrm{~cm}^{-3}$ (sample grown at $300^{\circ} \mathrm{C}$ ) and $4.0 \times 10^{12} \mathrm{~cm}^{-3}$ (sample grown at $400^{\circ} \mathrm{C}$ ). As previously published [22-24], the density of SAQDs can be estimated from the $\mathrm{C}-\mathrm{V}$ curves. Using the model described in [18], the density of SAQDs was found to be almost the same $\left(\sim 10^{12} \mathrm{~cm}^{-2}\right)$ for both samples. This result is in accordance with the Raman analysis presented in Figures 2(a) and 2(b), from where it can be noticed that relative intensities of Ge signal have the same order for both samples. Decreasing the temperature, the localization effects and consequently the presence of SAQDs can be readily observed: additional peaks (indicated by arrows) are observed in the capacitance signal because the dots are being filled and emptied following the applied voltage. Due to the low temperature, the electrons remain in the dots and their effects are now observed. In fact, from Figure 4(a), two peaks are observed at $150 \mathrm{~K}$ revealing the peaked character of the zero-dimensional density of states, while at room temperature only one-layer occupation is observed. Both samples (grown at $400^{\circ} \mathrm{C}$ and $300^{\circ} \mathrm{C}$ ) exhibit the same behavior. Our results are a clear indication of the presence of the quantum dots in the deepest layers of these samples. For the samples grown at the two highest temperatures, no clear signs of quantization could be observed with this technique.

\section{Conclusions}

The Raman spectra obtained with excitation energies near the resonance showed that the Ge optical phonons related to the quantum dots attain different wavenumbers as compared to the Ge bulk case. These wavenumber shifts are generated by the competition between two physical phenomena present in the structures: the strongest one is undoubtedly the compressive stress inside the SAQDs, but the phonon confinement effect is also relevant. The increase of the growth temperature implies in the nonhomogeneity of the vibrational modes, both due to the presence of large SAQDs - where the stress relaxation is more considerableand to the increase in the rate and depth of the Si diffusion along the growth direction.

The measurements with the variation of the excitation energy showed that the samples grown at 300,400, and $500^{\circ} \mathrm{C}$ present a size distribution of the SAQDs. However, it is clearly observed that this distribution is more heterogeneous in the second than in the first sample. Also, for the growth made at $500^{\circ} \mathrm{C}$, a regime of two modes can be inferred: the first one presenting dots with sizes comparable to those found for the lower temperatures and the second with much larger three-dimensional islands. Besides confirming the presence of the quantum dots grown at the two lowest temperatures, the $\mathrm{C}-\mathrm{V}$ measurements were able to estimate the electron densities and the quantum dot densities in the deepest layers of these samples.

The resonant Raman data led us to identify a principal electronic transition attributed to the Ge $E_{1}$ gap. A secondary maximum observed for the growth temperatures 400, 500, and $600^{\circ} \mathrm{C}$ was associated to the $\mathrm{Ge}_{x} \mathrm{Si}_{1-x}$ alloy formed in the structures and allowed us to conclude that the $\mathrm{Si}$ amount is negligible for the lowest growth temperature (where 


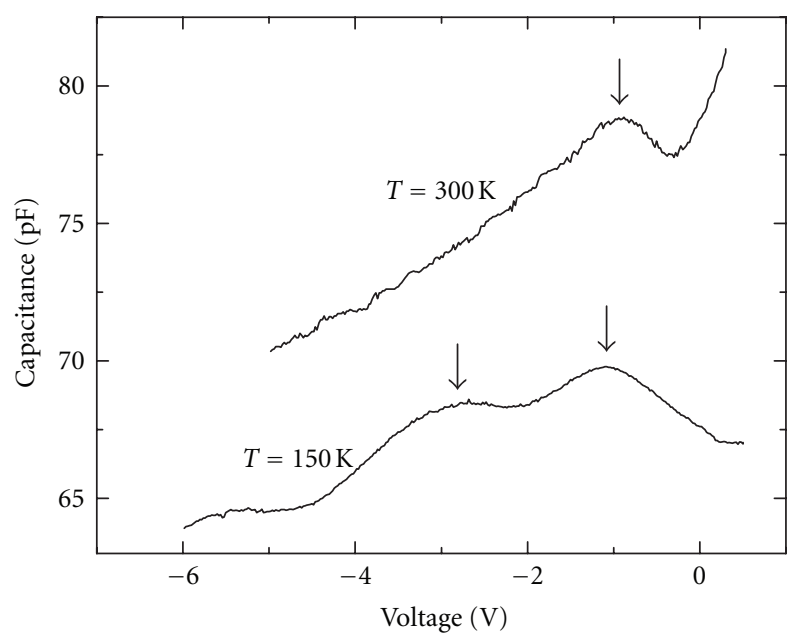

(a)

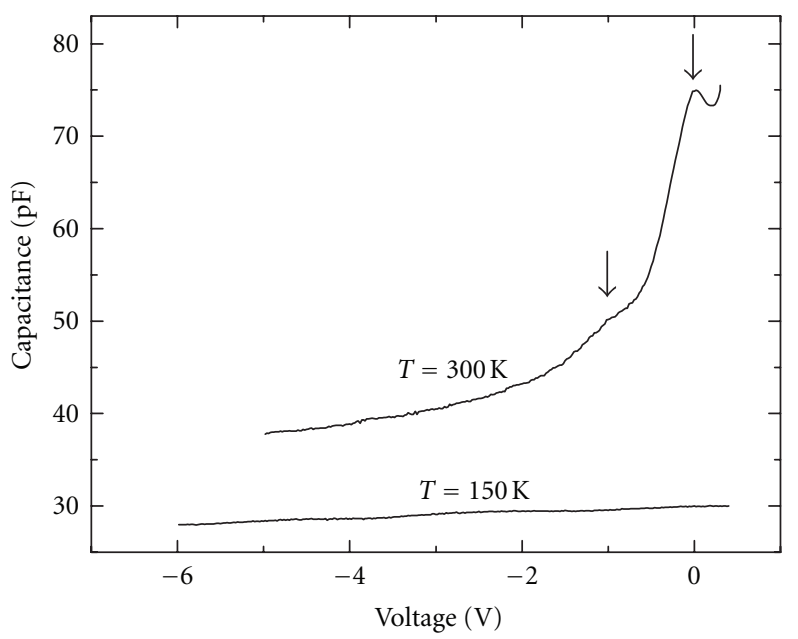

(b)

FIgURE 4: Capacitance versus Voltage measurements for the samples grown at (a) $300^{\circ} \mathrm{C}$ and (b) $400^{\circ} \mathrm{C}$.

the feature is not present), while for the highest temperature it reaches $50 \%$ of the alloy composition.

\section{Acknowlegments}

The authors are grateful to financial support from Brazilian agencies FAPESP and CNPq.

\section{References}

[1] K. L. Wang, J. L. Liu, and G. Jin, "Self-assembled Ge quantum dots on Si and their applications," Journal of Crystal Growth, vol. 237-239, no. 1-4, pp. 1892-1897, 2002.

[2] S. Banerjee, S. Nozaki, and H. Morisaki, "Coulomb-blockade effect observed at room temperature in Ge nanocrystalline films deposited by the cluster-beam evaporation technique," Applied Physics Letters, vol. 76, no. 4, pp. 445-447, 2000.

[3] W. K. Choi, W. K. Chim, C. L. Heng et al., "Observation of memory effect in germanium nanocrystals embedded in an amorphous silicon oxide matrix of a metal-insulatorsemiconductor structure," Applied Physics Letters, vol. 80, no. 11, pp. 2014-2016, 2002.

[4] Y. X. Jie, Y. N. Xiong, A. T. S. Wee, C. H. A. Huan, and W. Ji, "Dynamics of optical nonlinearity of Ge nanocrystals in a silica matrix," Applied Physics Letters, vol. 77, no. 24, pp. 39263928, 2000.

[5] J. L. Liu, G. Jin, Y. S. Tang, Y. H. Luo, K. L. Wang, and D. P. $\mathrm{Yu}$, "Optical and acoustic phonon modes in self-organized Ge quantum dot superlattices," Applied Physics Letters, vol. 76, no. 5, pp. 586-588, 2000.

[6] G. Matmon, D. J. Paul, L. Lever et al., "Si/SiGe quantum cascade superlattice designs for terahertz emission," Journal of Applied Physics, vol. 107, no. 5, Article ID 053109, 2010.

[7] V. A. Markov, H. H. Cheng, C. T. Chia et al., "RHEED studies of nucleation of $\mathrm{Ge}$ islands on $\mathrm{Si}(001)$ and optical properties of ultra-small Ge quantum dots," Thin Solid Films, vol. 369, no. 1, pp. 79-83, 2000.

[8] G. Capellini, M. De Seta, and F. Evangelisti, "SiGe intermixing in Ge/Si(100) islands," Applied Physics Letters, vol. 78, no. 3, pp. 303-305, 2001.
[9] M. W. Dashiell, U. Denker, C. Müller et al., "Photoluminescence of ultrasmall Ge quantum dots grown by molecularbeam epitaxy at low temperatures," Applied Physics Letters, vol. 80, no. 7, pp. 1279-1281, 2002.

[10] M. Brehm, M. Grydlik, H. Groiss et al., "The influence of a Si cap on self-organized SiGe islands and the underlying wetting layer," Journal of Applied Physics, vol. 109, no. 12, Article ID 123505, 2011.

[11] F. Ratto, F. Rosei, A. Locatelli et al., "Composition of $\mathrm{Ge}(\mathrm{Si})$ islands in the growth of Ge on Si(111)," Applied Physics Letters, vol. 84, no. 22, pp. 4526-4528, 2004.

[12] A. G. Milekhin, A. I. Nikiforov, M. Y. Ladanov et al., "Phonons in Ge/Si quantum dot structures: influence of growth temperature," Physica E, vol. 21, no. 2-4, pp. 464-468, 2004.

[13] M. I. Alonso and K. Winer, "Raman spectra of c- $\mathrm{Si}_{1-x} \mathrm{Ge}_{x}$ alloys," Physical Review B, vol. 39, no. 14, pp. 10056-10062, 1989.

[14] M. A. Renucci, J. B. Renucci, R. Zeyher, and M. Cardona, "Second-order Raman scattering in germanium in the vicinity of the $\mathrm{E}_{1}, \mathrm{E}_{1}+\Delta_{1}$ edges," Physical Review B, vol. 10, no. 10, pp. 4309-4323, 1974.

[15] S. H. Kwok, P. Y. Yu, C. H. Tung et al., "Confinement and electron-phonon interactions of the $\mathrm{E}_{1}$ exciton in selforganized Ge quantum dots," Physical Review B, vol. 59, no. 7, pp. 4980-4984, 1999.

[16] F. Cerdeira, A. Pinczuk, and J. C. Bean, "Observation of confined electronic states in $\mathrm{Ge}_{x} \mathrm{Si}_{1-x} \mathrm{Si}$ strained-layer superlattices," Physical Review B, vol. 31, no. 2, pp. 1202-1204, 1985.

[17] R. Schorer, G. Abstreiter, H. Kibbel, and H. Presting, "Resonant-Raman-scattering study on short-period Si/Ge superlattices," Physical Review B, vol. 50, no. 24, pp. 1821118218, 1994.

[18] A. G. Milekhin, D. Tenne, and D. R. T. Zahn(, "Quantum dot structures: raman and infrared spectroscopy," in Quantum Dots and Nanowires, S. Bandyopadhyay and H. S. Nalwa, Eds., pp. 375-419, American Scientific, California, Calif, USA, 2003.

[19] A. J. Chiquito, Y. A. Pusep, S. Mergulhão, and J. C. Galzerani, "Carrier confinement in an ultrathin barrier GaAs/AlAs superlattice probed by capacitance-voltage measurements," Physica E, vol. 13, no. 1, pp. 36-42, 2002. 
[20] S. M. Sze, Physics of Semiconductors Devices, John Wiley and Sons, New York, NY, USA, 1981.

[21] A. J. Chiquito, Y. A. Pusep, S. Mergulhão, J. C. Galzerani, and N. T. Moshegov, "Effect of photogenerated holes on capacitance-voltage measurements in InAs/GaAs selfassembled quantum dots," Physical Review B, vol. 61, no. 7, pp. 4481-4484, 2000.

[22] A. I. Yakimov, A. V. Dvurechenskii, A. I. Nikiforov, and O. P. Pchelyakov, "Formation of zero-dimensional hole states in Ge/Si heterostructures probed with capacitance spectroscopy," Thin Solid Films, vol. 336, no. 1-2, pp. 332-335, 1998.

[23] A. J. Chiquito and M. G. de Souza, "Temperature dependence of the electron distribution in a GaAs matrix with embedded InAs quantum dots," Physica E, vol. 25, no. 4, pp. 613-618, 2005.

[24] A. J. Chiquito, Y. A. Pusep, S. Mergulhão, J. C. Galzerani, and N. T. Moshegov, "Capacitance-voltage profile in a structure with negative differential capacitance caused by the presence of InAs/GaAs self-assembled quantum dots," Physical Review $B$, vol. 61, no. 8, pp. 5499-5504, 2000. 

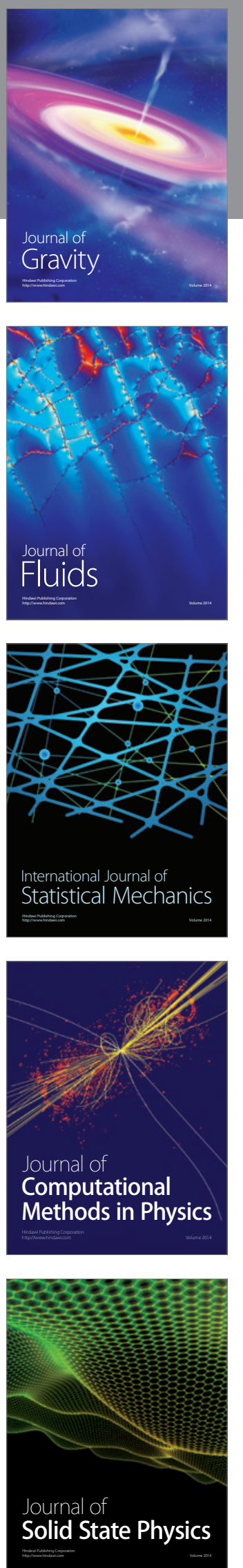

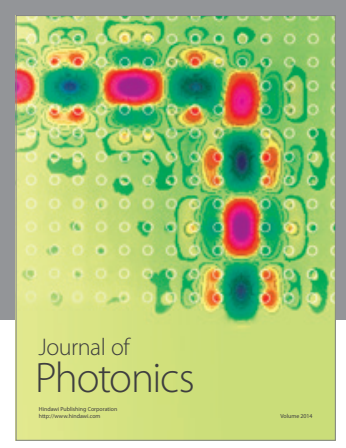

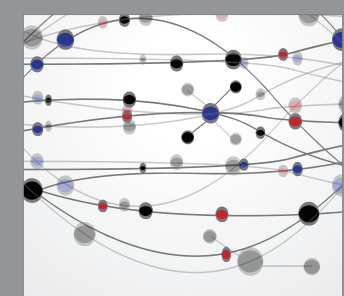

The Scientific World Journal
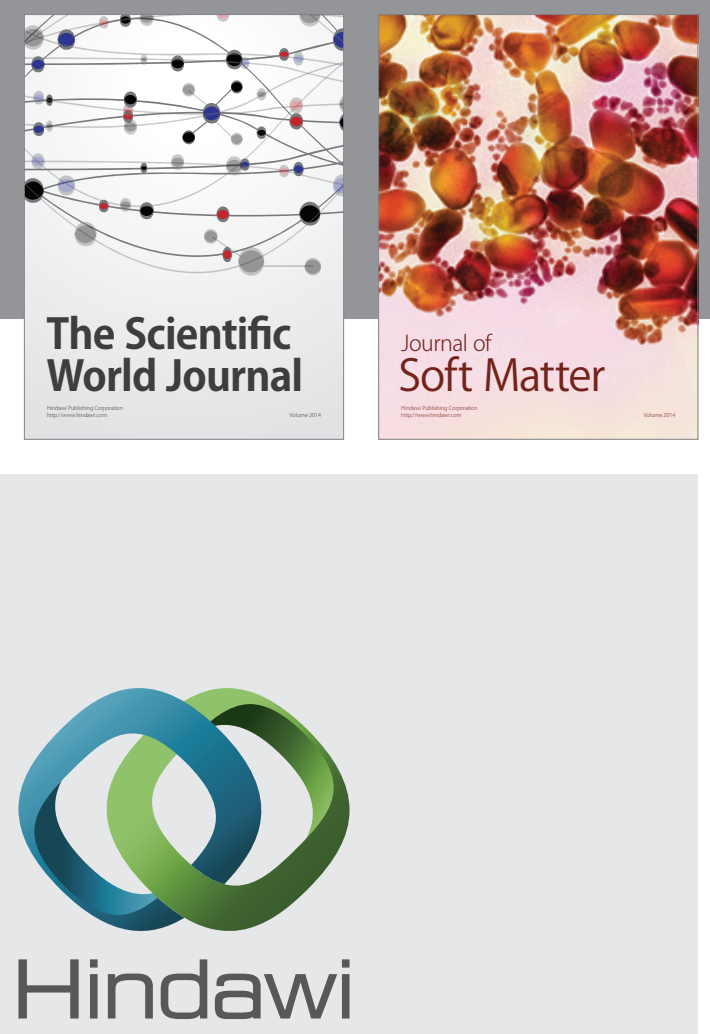

Submit your manuscripts at

http://www.hindawi.com
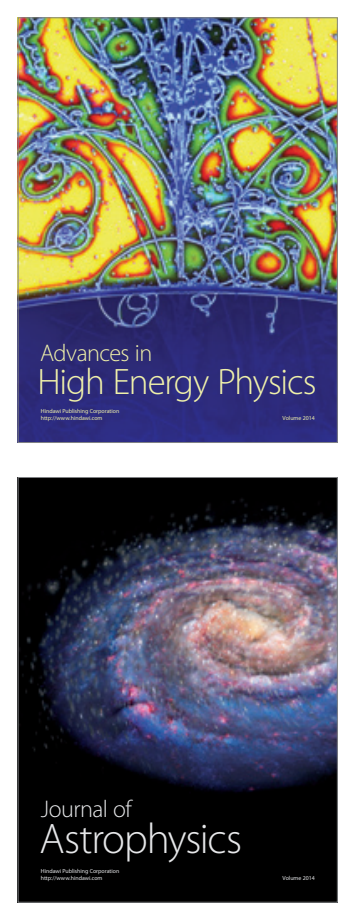
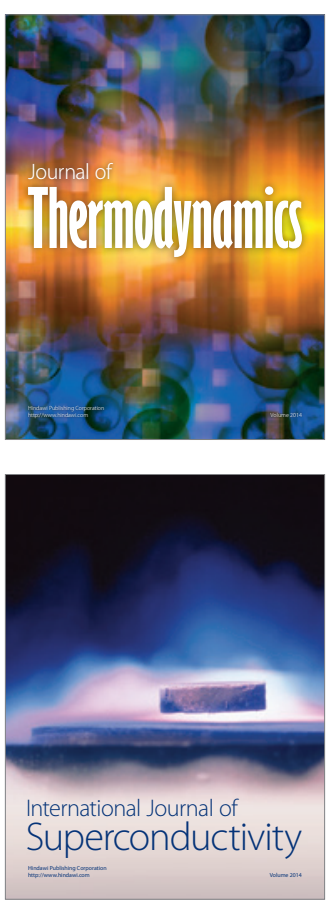
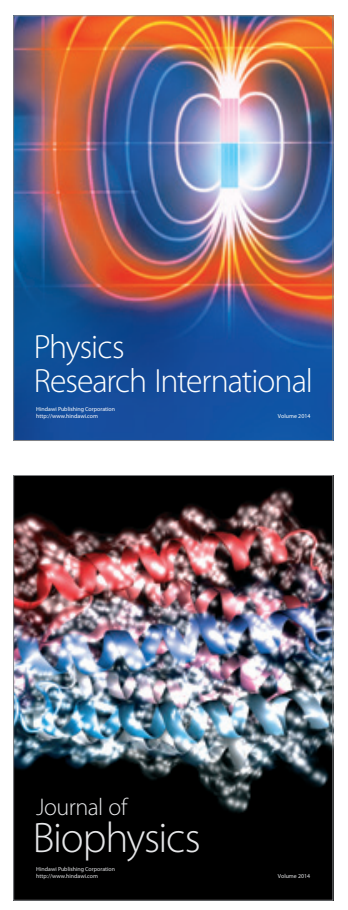
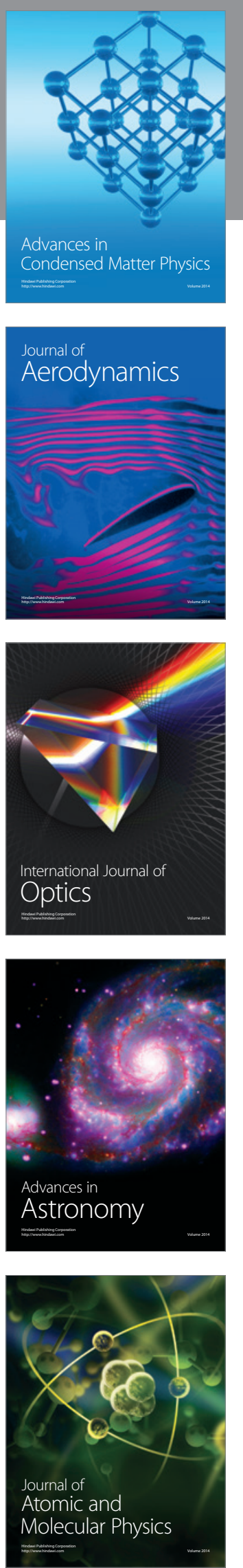